PROCEEDINGS OF THE

AMERICAN MATHEMATICAL SOCIETY

Volume 134, Number 9, September 2006, Pages 2613-2620

S 0002-9939(06)08255-4

Article electronically published on February 17, 2006

\title{
SUPER-ERGODIC OPERATORS
}

\author{
M. YAHDI
}

(Communicated by Joseph A. Ball)

\begin{abstract}
The aim of this work is to study operators naturally connected to Ergodic operators in infinite-dimensional Banach spaces, such as UniformErgodic, Cesaro-bounded and Power-bounded operators, as well as stable and superstable operators. In particular, super-Ergodic operators are introduced and shown to be strictly between Ergodic and Uniform-Ergodic operators, and that any power bounded operator is super-Ergodic in a superreflexive space. New relationships between these operators are shown, others are proven to be optimal or can be ameliorated according to structural properties of the Banach space, such as the superreflexivity or with unconditional basis.
\end{abstract}

\section{INTRODUCTION}

For an infinite Banach space $X$ and a bounded operator $T$ on $X$, consider the sequence $\left\{A_{n}\right\}_{x \geq 1}$ of Cesaro-means of $T$ defined as follows:

$$
A_{n}=\frac{1}{n} \sum_{k=0}^{n-1} T^{k}, \quad \text { where } \quad T^{k}(x)=T\left(T^{k-1}(x)\right) .
$$

Definitions 1.1. An operator $T$ on a Banach space $X$ is

- Ergodic if the sequence $\left\{A_{n}\right\}_{x \geq 1}$ of Cesaro-means converges in the space of operators $L(X)$ equipped with the strong operator topology $S_{\text {op }}$, i.e. $\forall x \in$ $X,\left\{A_{n}(x)\right\}_{x \geq 1}\|\cdot\|$-converges in $X$.

- Uniformly Ergodic if the sequence $\left\{A_{n}\right\}_{x \geq 1}$ converges in $L(X)$ equipped with its natural norm.

- Weakly Ergodic if for any $x \in X$, the sequence $\left\{A_{n}(x)\right\}_{x \geq 1}$ weakly converges in $X$.

- Cesaro-bounded if the norms of $\left\{A_{n}\right\}_{n}$ are uniformly bounded.

- Power-bounded if the norms of $\left\{T^{n}\right\}_{n}$ are uniformly bounded.

In section 3, "super-Ergodic" operators are introduced, followed by a complete investigation of all possible relationships between all the operators above as well as stable and superstable operators (see [Y2]). Counter examples are given to show that some known or new results are optimal. It is also shown when structures of the Banach space (reflexivity, superreflexivity, with special complemented subspaces) affect or not the relationships between these operators.

Received by the editors March 11, 2005 and, in revised form, March 23, 2005.

2000 Mathematics Subject Classification. Primary 47A35, 46B08; Secondary 47B07, 47B99.

Key words and phrases. Super-Ergodic, Ergodic operator, stable operator, Banach space, ultrapower.

(C)2006 American Mathematical Society Reverts to public domain 28 years from publication 
Throughout this work, unless it is said otherwise, $X$ denotes an infinite-dimensional Banach space with a norm $\|\cdot\|$, and $B_{X}=\{x \in X:\|x\| \leq 1\}$ denotes its closed unit ball.

The space of bounded operators on $X$ is noted by $L(X)$, and $T$ denotes a typical element of such a space. A norm in $L(X)$ is given by $\|T\|:=\sup _{x \in B_{X}}\|T x\|$, and the strong operator topology is noted $S_{o p}$.

Recall that for a bounded operator $T$ in a Banach space $X$, the spectrum of $T$ is define by $\sigma(T)=\{\lambda \in \mathbb{C}:(T-\lambda I)$ is not invertible $\}$, and the spectral radius $r(T)$ of $T$, satisfies

$$
r(T):=\limsup _{n \rightarrow \infty}\left\|T^{n}\right\|^{\frac{1}{n}}=\max \{|\lambda|: \lambda \in \sigma(T)\} .
$$

$\mathbb{N}$ is the set of positive integers and $\left\{a_{n}\right\}_{n}$ denotes a countable sequence with $n \in \mathbb{N}$. See $[\mathrm{DS}],[\mathrm{Ru}],[\mathrm{Ro}]$ or $[\mathrm{Wo}]$ for other definitions.

\section{RELATIONSHIPS BETWEEN OPERATORS RELATED TO ERGODICITY}

It is obvious that "uniformly Ergodic" implies "Ergodic", and that "Ergodic" implies "weakly Ergodic". We will see when these implications are strict or when equivalences are possible. We will also consider Cesaro and power-bounded operators. But first, we have the following.

Remarks 2.1.

- If $T$ is a bounded operator with spectral radius $r(T)<1$, then $T$ is uniformly Ergodic because there exists $0<\alpha<1$ and a positive integer $N$ such that $\left\|T^{n}\right\| \leq \alpha^{n}$ for all $n \geq N$.

- Since $\frac{1}{n} T^{n}=\frac{n+1}{n} A_{n+1}-A_{n}$, then:

$$
\begin{aligned}
& T \text { is Ergodic } \Longrightarrow \lim _{n \rightarrow \infty} \frac{1}{n}\left\|T^{n} x\right\|=0 \quad \forall x \in X . \\
& T \text { is uniformly Ergodic } \Longrightarrow \lim _{n \rightarrow \infty} \frac{1}{n}\left\|T^{n}\right\|=0 .
\end{aligned}
$$

By Banach-Steinhaus, it follows from (2.0.1) that any Ergodic operator is Cesarobounded. Clearly a power-bounded operator is Cesaro-bounded. However, both implications are strict. Indeed, when an operator is power bounded or Ergodic we have $\lim _{n \rightarrow \infty} \frac{1}{n}\left\|T^{n} x\right\|=0, \quad \forall x \in X$, which fails for the Cesaro-bounded operator (given by Assani [As] $T=\left(\begin{array}{cc}-1 & 2 \\ 0 & -1\end{array}\right)$, with $X=\mathbb{R}^{2}$ and $x=(0,1) \in \mathbb{R}^{2}$.

From the Mean Ergodic Theorem (see $[\mathrm{Kr}$ or $[\mathrm{E}]$ ), we deduce that for a powerbounded operator $T$ on a Banach space $X$, the Ergodicity of $T$ is equivalent to the weak Ergodicity of $T$, and in particular that for all $x \in X,\left\{A_{n} x\right\}_{n \in \mathbb{N}}$ has a weak accumulation point. Since every norm-bounded set in a reflexive Banach space is weakly relatively compact, the following theorem (of Yosida-Kakutani) follows (also proved independently by E. Lorch; see $[\mathrm{L}]$ or $[\mathrm{Kr}$, p. 73).

Theorem 2.2. Any power-bounded operator on a reflexive Banach space $X$ is Ergodic.

The reflexivity is necessary in this theorem as shown below. 
Example 2.3. Let $X=\mathcal{C}([0,1])$ be the space of continuous functions on the interval $[0,1]$ equipped with the norm $\|f\|_{\infty}=\sup _{x \in[0,1]}|f(x)|$.

Let $M$ be the operator of multiplication defined for all $f \in X$ by

$$
M f(t)=t . f(t) \quad \text { for all } t \in[0,1] .
$$

For any positive integer $n$ we have $M^{n} f(t)=t^{n} \cdot f(t)$, thus $\left\|M^{n}\right\| \leq 1$. Therefore, $M$ is a power-bounded operator. On the other hand, for any continuous function $f \in X$ and any real $t \in[0,1)$ we have

$$
\begin{aligned}
\left\|A_{n} f-A_{2 n} f\right\|_{\infty} & \geq|f(t)|\left[\frac{1}{n} \sum_{k=0}^{n-1} t^{k}-\frac{1}{2 n} \sum_{k=0}^{2 n-1} t^{k}\right] \\
& =\frac{|f(t)|}{2 n}\left[\frac{2\left(1-t^{n}\right)-\left(1-t^{2 n}\right)}{1-t}\right] \\
& =\frac{|f(t)|}{2 n} \frac{\left(1-t^{n}\right)^{2}}{1-t} .
\end{aligned}
$$

By replacing $t$ by $t_{n}=1-\frac{1}{n}$, and since $\lim _{n \rightarrow \infty}\left(t_{n}\right)^{n}=\frac{1}{e}$, we get

$$
\lim _{n \rightarrow \infty}\left\|A_{n} f-A_{2 n} f\right\|_{\infty} \geq \lim _{n \rightarrow \infty} \frac{\left|f\left(t_{n}\right)\right|}{2} \cdot\left(1-\left(t_{n}\right)^{n}\right)^{2} \geq \frac{\left(1-\frac{1}{e}\right)^{2}}{2}|f(1)| .
$$

Hence, if $f$ is chosen such that $f(1) \neq 0$, the sequence $\left\{A_{n} f\right\}_{n \in \mathbb{N}}$ is not Cauchy in $X$. Therefore the operator $M$ is not Ergodic.

The next example shows that in Theorem 2.2, "Ergodic" cannot be replaced by "Uniformly Ergodic", even in a superreflexive space. Recall that a Banach space $X$ is superreflexive if any Banach space that is finitely representable in $X$ is reflexive, or equivalently if $X$ has an equivalent uniformly convex norm.

Example 2.4. Let $\left\{\lambda_{n}\right\}_{n \in \mathbb{N}}$ be a sequence of real numbers such that $\lim _{n \rightarrow \infty} \lambda_{n}=1$ and $\left|\lambda_{n}\right|<1$ for all $n \in \mathbb{N}$. Consider the multiplication operator defined on the superreflexive space $\ell_{2}(\mathbb{N})$ by

$$
T:\left(x_{n}\right)_{n \in \mathbb{N}} \longmapsto\left(\lambda_{n} x_{n}\right)_{n \in \mathbb{N}} .
$$

It is clear that $T$ is a contraction, and thus Ergodic (by Theorem 2.2). Let $A_{n}=$ $\frac{1}{n} \sum_{k=0}^{n-1} T^{k}$, and let $\left\{e_{i}\right\}_{i \in \mathbb{N}}$ be the canonical basis of $\ell_{2}(\mathbb{N})$. For any positive integers $n$ and $i$, we have

$$
\left\|A_{n} e_{i}\right\|_{2}=\left\|\frac{1}{n}\left(\lambda_{i}{ }^{0}+\lambda_{i}{ }^{1}+\cdots+\lambda_{i}{ }^{n-1}\right) e_{i}\right\|_{2} \leq \frac{1}{n} \frac{1}{1-\lambda_{i}} .
$$

Thus, $\lim _{n \rightarrow \infty}\left\|A_{n} e_{i}\right\|_{2}=0$ for all $i \in \mathbb{N}$. By equicontinuity, it follows that

$$
\lim _{n \rightarrow \infty}\left\|A_{n} x\right\|_{2}=0 \quad \forall x \in \ell_{2}(\mathbb{N}) .
$$

Assume now that $T$ is Uniformly Ergodic. Then necessarily $A_{n}$ converges uniformly to 0 . But, with $\lambda_{n}=1-\frac{1}{n}$ we get

$$
\lim _{n \rightarrow \infty}\left\|A_{n} e_{n}\right\|_{2}=\lim _{n \rightarrow \infty} \frac{1}{n} \frac{1-\left(1-\frac{1}{n}\right)^{n}}{\frac{1}{n}}=\lim _{n \rightarrow \infty} 1-\left(1-\frac{1}{n}\right)^{n}=1-\frac{1}{e} \neq 0 .
$$




\section{Super-ERgodic operators}

Since we cannot get a Uniformly Ergodic operator if we replace reflexive by superreflexive in Theorem 2.2, it is natural to investigate the existence of operators strictly stronger than Ergodic ones (and of course weaker than Uniformly Ergodic). In this section, super-Ergodic operators are introduced, their relationships with all previous operators are investigated, and examples are given to show that the results are optimal. First, we needed to introduce a "super-property" (see $\mathrm{He}$ ).

For a Banach space $X$ and an ultrafilter $\mathcal{U}$ on $\mathbb{N}$, we denote by

- $\ell^{\infty}(X)$ the Banach space of bounded sequences in $X$ and $\mathcal{C}_{\mathcal{U}}(X)$ its subspace of sequences $\left\{x_{n}\right\}_{n}$ such that $\lim _{\mathcal{U}}\left\|x_{n}\right\|=0$.

- $X_{\mathcal{U}}:=\ell^{\infty}(X) / \mathcal{C}_{\mathcal{U}}(X)$ the quotient space, called the $\mathcal{U}$-ultrapower of $X$, equipped with the canonical norm

$$
\|\bar{x}\|=\mathcal{U}-\lim _{n}\left\|x_{n}\right\| \quad \text { for any }\left\{x_{n}\right\}_{n} \in \bar{x}:=\left\{x_{n}\right\}_{n}+\mathcal{C}_{\mathcal{U}}(X) .
$$

- $T_{\mathcal{U}}$ is the ultrapower operator of $T$ defined on $X_{\mathcal{U}}$ by

$$
T_{\mathcal{U}}(\bar{x}):=\left\{T x_{n}\right\}_{n}+\mathcal{C}_{\mathcal{U}}(X) \quad \text { for any }\left\{x_{n}\right\}_{n} \in \bar{x}:=\left\{x_{n}\right\}_{n}+\mathcal{C}_{\mathcal{U}}(X)
$$

Definition 3.1. An operator $T$ on a Banach space $X$ is super-Ergodic if for any ultrafilter $\mathcal{U}$ on $\mathbb{N}$, the operator $T_{\mathcal{U}}$ is Ergodic on $X_{\mathcal{U}}$.

The following lemma simplifies the connection between the Cesaro-means and the ultrapowers.

Lemma 3.2. The ultrapower of a Cesaro-mean is the Cesaro-mean of the ultrapower $\left(A_{n}\right)_{\mathcal{U}}=\left(A_{\mathcal{U}}\right)_{n}$, noted $A_{n}^{\mathcal{U}}$.

Proof. For any $\bar{x}=\left(x_{p}\right)_{p \in \mathbb{N}}+\mathcal{C}_{\mathcal{U}}(X)$ in $X_{\mathcal{U}}$ and any $n \in \mathbb{N}$, we have

$$
\begin{gathered}
\left(A_{n}\right)_{\mathcal{U}}(\bar{x})=\left(\frac{1}{n} \sum_{k=0}^{n-1} T^{k} x_{p}\right)_{p}+\mathcal{C}_{\mathcal{U}}(X)=\frac{1}{n} \sum_{k=0}^{n-1}\left[\left(T^{k} x_{p}\right)_{p}+\mathcal{C}_{\mathcal{U}}(X)\right] \\
\left(A_{\mathcal{U}}\right)^{n}(\bar{x})=\frac{1}{n} \sum_{k=0}^{n-1} T_{\mathcal{U}}^{k}(\bar{x})=\frac{1}{n} \sum_{k=0}^{n-1}\left[\left(T^{k} x_{p}\right)_{p}+\mathcal{C}_{\mathcal{U}}(X)\right]
\end{gathered}
$$

Clearly a super-Ergodic operator is Ergodic; Example 3.3 below shows that this implication is strict. But first recall that an operator $T$ is stable if each of its orbits $\mathcal{O}_{T}(x):=\left\{T^{n}(x): n \in \mathbb{N}\right\}$ is relatively compact for every $x \in X$, and $T$ is superstable if for any ultrafilter $\mathcal{U}$ on $\mathbb{N}$, the ultrapower $T_{\mathcal{U}}$ is stable on $X_{\mathcal{U}}$ (see Y2]).

Example 3.3. Let $X=\ell^{1}(\mathbb{N})$ and $S$ be the left-shift operator on $X$. Let $\left\{e_{n}\right\}_{n \in \mathbb{N}}$ be the canonical basis of $X$. This operator is clearly Ergodic since $\lim _{n \rightarrow \infty}\left\|S^{n} x\right\|=$ $0, \forall x \in X$ (it is even stable; see YY2]). However, $S$ is not super-Ergodic. Indeed, let $\mathcal{U}$ be an ultrafilter on $\mathbb{N}$, and $A_{n}^{\mathcal{U}}$ the $n^{\text {th }}$ Cesaro-mean of $S_{\mathcal{U}}$. Let $\bar{e} \in X_{\mathcal{U}}$, the 
class of $\left(e_{k}\right)_{k \in \mathbb{N}}$. We have

$$
\begin{aligned}
\left\|A_{n}^{\mathcal{U}}(\bar{e})-A_{2 n}^{\mathcal{U}}(\bar{e})\right\|_{X_{\mathcal{U}}} & =\mathcal{U}-\lim _{k}\left\|A_{n}\left(e_{k}\right)-A_{2 n}\left(e_{k}\right)\right\|_{X} \\
& =\mathcal{U}-\lim _{k}\left\|\frac{1}{n} \sum_{i=0}^{n-1} e_{k+i}-\frac{1}{2 n} \sum_{i=0}^{2 n-1} e_{k+i}\right\|_{1} \\
& =\mathcal{U}-\lim _{k}\left\|\frac{1}{2 n} \sum_{i=0}^{n-1} e_{k+i}-\frac{1}{2 n} \sum_{i=n}^{2 n-1} e_{k+i}\right\|_{1} \\
& =\mathcal{U}-\lim _{k}\left(\frac{1}{2 n} \cdot n+\frac{1}{2 n} \cdot n\right) \\
& =1 .
\end{aligned}
$$

So, $\left\{A_{n}^{\mathcal{U}}(\bar{e})\right\}_{n \in \mathbb{N}}$ is not Cauchy, and thus $S$ is not super-Ergodic.

Using this example and relationships between stable and superstable operators given in [Y2, we have the following corollary.

Corollary 3.4. For any Banach space $X$, a stable operator is Ergodic, and a superstable operator is super-Ergodic. However, if $X$ contains a complemented $\ell^{1}(\mathbb{N})$, then it has a stable but not super-Ergodic operator.

Since for any operator $T$, any ultrafilter $\mathcal{U}$ and any positive integer $n$, we have $\left\|T_{\mathcal{U}}^{n}\right\| \leq\left\|T^{n}\right\|$, it follows from Theorem 2.2 that:

Theorem 3.5. Any power-bounded operator on a superreflexive Banach space is super-Ergodic.

It is without surprise that we have the following result.

Proposition 3.6. The uniform Ergodicity is a super-property.

Proof. Let $T$ be a uniformly Ergodic operator on a Banach space $X$. Let $\mathcal{U}$ be an ultrafilter on $\mathbb{N}$. Note by $\left\{A_{n}\right\}_{n \geq 1}$ and $\left\{A_{n \geq 1}^{\mathcal{U}}\right\}_{n}$ the Cesaro-means associated with $T$ and $T_{\mathcal{U}}$. By hypothesis, the sequence $\left\{A_{n}\right\}_{n \geq 1}$ converges in $L(X)$ to an operator noted $A$. Let $A^{\mathcal{U}}$ be the ultrapower operator of $A$. Let $\bar{x}=\left(x_{k}\right)_{k \in \mathbb{N}}+\mathcal{C}_{\mathcal{U}}(X) \in X_{\mathcal{U}}$. We have

$$
\begin{aligned}
\left\|\left(A_{n}^{\mathcal{U}}-A^{\mathcal{U}}\right)(\bar{x})\right\|_{X_{\mathcal{U}}} & =\mathcal{U}-\lim _{k}\left\|A_{n} x_{k}-A x_{k}\right\| \\
& \leq \mathcal{U}-\lim _{k}\left\|A_{n}-A\right\|\left\|x_{k}\right\| \\
& =\left\|A_{n}-A\right\| \mathcal{U}-\lim _{k}\left\|x_{k}\right\| \\
& \leq\left\|A_{n}-A\right\| .
\end{aligned}
$$

Therefore $\left\|A_{n}^{\mathcal{U}}-A^{\mathcal{U}}\right\| \leq\left\|A_{n}-A\right\|$. Thus, $\left\{A_{n}^{\mathcal{U}}\right\}_{n \geq 1}$ norm-converges in $L\left(X_{\mathcal{U}}\right)$ to the operator $A^{\mathcal{U}}$. In other terms, $T_{\mathcal{U}}$ is uniformly Ergodic.

Corollary 3.7. Any uniformly Ergodic operator is super-Ergodic.

Proof. Clearly because a uniformly Ergodic operator is Ergodic.

Most of the results in this section are optimal. In Corollary 3.4 we saw a condition that shows that "super-Ergodic" is strictly stronger than "Ergodic". Actually it is the case even in a reflexive space as show in Example 3.8 below. In addition, this example shows that Theorem 3.5 fails in the absence of the superreflexivity. 
On the other hand, a super-Ergodic operator may not be superstable. In fact even a uniformly Ergodic operator may not be stable even in a superreflexive space, as shown in Example 3.9.

Example 3.8. Let $X=\ell^{p}\left(\ell_{n}^{\infty}\right)$; the $\ell^{p}$-sum of the spaces $\ell_{n}^{\infty}, n \in \mathbb{N}$, where $1<p<\infty$. $X$ is reflexive but not superreflexive. Let $\left(\alpha_{n}\right)_{n \in \mathbb{N}}$ be a sequence strictly increasing in $[0,1)$ that converges to 1 . For each positive integer $n$, let $T_{n}\left(\xi_{1}, \ldots, \xi_{n}\right)=\left(\alpha_{1} \xi_{1}, \ldots, \alpha_{n} \xi_{n}\right) \in \ell_{n}^{\infty}$. Consider

$$
\begin{aligned}
T: \ell^{p}\left(\ell_{n}^{\infty}\right) & \longrightarrow \ell^{p}\left(\ell_{n}^{\infty}\right), \\
\left(x_{n}\right)_{n \in \mathbb{N}^{*}} & \longmapsto\left(T_{n} x_{n}\right)_{n \in \mathbb{N}^{*}} .
\end{aligned}
$$

$T$ is a power-bounded operator since it is a contraction. Let

$$
Z_{m}=\left(z_{m, n}\right)_{n \in \mathbb{N}} \in \ell^{p}\left(\ell_{n}^{\infty}\right) \quad \text { with }\left\{\begin{array}{l}
z_{m, m}=(1, \ldots, 1) \in \ell_{m}^{\infty}, \\
z_{m, n}=0 \text { if } n \neq m .
\end{array}\right.
$$

Let $Z$ be the class of $\left(Z_{m}\right)_{m}$ in $X_{\mathcal{U}}$ for $\mathcal{U}$ a non-trivial ultrafilter on $\mathbb{N}$. Let $A_{N}$ (resp. $A_{N}^{\mathcal{U}}$ ) be the $N^{\text {th }}$ Cesaro-mean of $T$ (resp. $T_{\mathcal{U}}$ ). We have

$$
\begin{aligned}
A_{N}\left(Z_{m}\right) & =\frac{1}{N} \sum_{k=0}^{N-1} T^{k}\left(Z_{m}\right) \\
& =\frac{1}{N} \sum_{k=0}^{N-1}\left(T_{n}^{k} z_{m, n}\right)_{n \in \mathbb{N}} \\
& =\frac{1}{N}(0, \ldots, 0, \overbrace{\sum_{k=0}^{N-1}\left(\alpha_{1}^{k}, \ldots, \alpha_{m}^{k}\right)}^{m^{t h} \operatorname{term}}, 0, \ldots) \\
& =\left(0, \ldots, 0, \frac{1}{N}\left(\frac{1-\alpha_{1}^{N}}{1-\alpha_{1}}, \ldots, \frac{1-\alpha_{m}^{N}}{1-\alpha_{m}}\right), 0, \ldots\right) .
\end{aligned}
$$

Therefore,

$$
\left\|A_{N}\left(Z_{m}\right)-A_{2 N}\left(Z_{m}\right)\right\|_{X}=\frac{1}{2 N}\left\|\left(\frac{\left(1-\alpha_{1}^{N}\right)^{2}}{1-\alpha_{1}}, \ldots, \frac{\left(1-\alpha_{m}^{N}\right)^{2}}{1-\alpha_{m}}\right)\right\|_{\ell_{m}^{\infty}} .
$$

With a choice of $\alpha_{n}=1-\frac{1}{n}$, we get for all $m \geq N$

$$
\left\|A_{N}\left(Z_{m}\right)-A_{2 N}\left(Z_{m}\right)\right\| \geq \frac{\left(1-\alpha_{N}^{N}\right)^{2}}{2} .
$$

Since $\mathcal{U}$ is a non-trivial ultrafilter, it follows that for any $N \in \mathbb{N}$,

$$
\left\|A_{N}^{\mathcal{U}}(Z)-A_{2 N}^{\mathcal{U}}(Z)\right\|=\mathcal{U}-\lim _{m}\left\|A_{N}\left(Z_{m}\right)-A_{2 N}\left(Z_{m}\right)\right\| \geq \frac{\left(1-\alpha_{N}^{N}\right)^{2}}{2} .
$$

Therefore, $\lim _{N \rightarrow \infty}\left\|A_{N}^{\mathcal{U}}(Z)-A_{2 N}^{\mathcal{U}}(Z)\right\| \geq \frac{\left(1-\frac{1}{e}\right)^{2}}{2}>0$. Thus the sequence $\left\{A_{N}^{\mathcal{U}}(Z)\right\}_{N}$ is not Cauchy, and $M$ is not super-Ergodic.

Example 3.9. Let $X=L^{2}(\Lambda, \mu)$, where $\Lambda$ is an uncountable and closed subset of the unit circle $\mathbb{T}$ such that $1 \notin \Lambda$ and $\mu$ is a diffuse measure with support $\Lambda$ 
( $\mu$ exists; see $[\mathrm{Sc}, 19.7 .6$ and 8.5.5). $X$ is supereflexive. Define the operator of multiplication $M$ on $X$ by

$$
M f(\lambda)=\lambda f(\lambda) \quad \forall f \in X \text { and } \forall \lambda \in \Lambda .
$$

$M$ is an isometry and thus a power-bounded operator, so $M$ is super-Ergodic (Theorem [3.5). On the other hand, the spectrum of $M$ is $\sigma(M)=\Lambda$. Thus, $M$ is uniformly Ergodic since $1 \notin \sigma(M)$ (by the Theorem on page 90 in $[\mathrm{Kr}$ ). But $M$ is not stable. Indeed, first $M$ does not have any eigenvalue because $\mu$ is a diffuse measure. From the theorem of decomposition of Glicksberg-De Leeuw ( $\mathrm{Kr}] 2-4-4$, $2-4-5)$ or $[\mathrm{NR}]$ ), it follows that

$$
X=X_{w}=\left\{x \in X ; 0 \text { is a weak-point of accumulation in } \mathcal{O}_{M}(x)\right\} .
$$

By Corollary 1.4 in $\mathbf{N R}$, it follows that $M$ is stable if and only if $X=\{x \in$ $\left.X ; \lim _{n \rightarrow \infty}\left\|M^{n} x\right\|=0\right\}$, which means $X=\{0\}$ since $M$ is an isometry. Hence, $M$ cannot be stable.

A natural question is to try to see if there is a general property of the space that can place a strong limit on what sort of alternative relationships between those operators might be true, thus eliminating not just one conjectured equivalence, but many at one stroke. However, with the difficulty of the description of super-Ergodic operators, this leads to an analogy with questions in Banach space theory concerning the existence or the relationships between mathematical objects according to the structure of the space of study. Translating these mathematical concepts to families of sets, then examining their positions in the descriptive set hierarchy has proved to be a productive approach (see [Y1] and Y2]). Moreover, application of descriptive set theory produces dichotomy theorems. Rosenthal's $\ell_{1}$-theorem in [R] and a theorem from Gowers in $[\mathrm{G}$ ] are examples of such a theorem. An example in $\mathrm{Y} 2$ is about the set of superstable operators, where it is shown that its complexity is connected to whether the space has some kind of unconditional basis or whether it is hereditarily indecomposable. It would be interesting to use similar techniques for super-Ergodic operators (investigated in a future paper).

\section{REFERENCES}

[As] I. Assani, Operators with bounded powers and the pointwise Ergodic theorem in $L^{p}[0,1]$, (French), Canad. J. Math. 38, (1986), No. 4, 937-946. MR0854147(87k:47015)

[DS] N. Dunford, J. Schwartz, Linear operator, Part. I, A Wiley-Interscience Publication, New York-London-Sydney (1988). MR.1009162 (90g:47001a)

[E] R. Emilion, Mean-bounded operators and mean Ergodic theorems, J. Funct. Anal. 61, (1985), No. 1, 1-14. MR0779737 (86h:47009)

[G] W. T. Gowers, An infinite Ramsey theorem and some Banach-space dichotomies, Annals of Math. 156, (2002), No. 3, 797-833. MR1954235 (2005a:46032)

[He] S. Heinrich, Ultraproducts in Banach space theory, J. Reine Angew. Math. 313, (1980), 72-104. MR0552464 (82b:46013)

[Kr] U. Krengel, Ergodic theorems, Walter de Gruyter, Berlin-New York (1985). MR0797411 (87i:28001)

[L] Edgar R. Lorch, Means of iterated transformatios in reflexive vector spaces, Bull. AMS 45, (1939), 945-947. MR0001460 (1:242e)

[NR] R. Nagel, F. Räbiger, Superstable operators on Banach spaces, Israel J. Math. 81, (1993), No. 1-2, 213-226. MR1231188 (94i:47006)

[R] H. Rosenthal, A characterization of Banach spaces containing $\ell_{1}$, Proc. Nat. Acad. Sci. U.S.A. 71, (1974), 2411-2413. MR0358307 (50:10773)

[Ro] H.L. Royden, Real Analysis, Macmillan, New York, 1963. MR0151555 (27:1540) 
[Ru] W. Rudin, Functional Analysis, McGraw, New York, 1973. MR0365062 (51:1315)

[Sc] H.H. Schaefer, Banach lattice and Positive operators, Grundl. Math. Wiss. 215, SpringerVerlag, New York-Heindelberg-Berlin (1974). MR0423039 (54:11023)

[Wo] P. Wojtaszczyk, Banach spaces for Analysts, Cambridge Studies in Advanced Math., 25 (1991). MR1144277 (93d:46001)

[Y1] M. Yahdi, The topological complexity of sets of convex differentiable functions, Revista Matematica Complutense, Vol. 11, no. 1, 79-91, 1998. MR.1634617 (99d:54017)

[Y2] M. Yahdi, The Spectrum of a Super-stable Operator and co-analytic families of operators, Illinois Journal of Mathematics 45, no. 1 (2001), 91-111. MR1849987 (2002f:47007)

Department of Mathematics and Computer Science, Ursinus College, Collegeville, Pennsylvania 19426

E-mail address: myahdi@ursinus.edu 